\title{
Comparação das Variáveis Cardiorrespiratórias de Adolescentes Obesos e não Obesos em Esteira e Bicicleta Ergométrica
}

\section{Comparison of the Cardiorespiratory Variables of Obese and Non-Obese Adolescents on Treadmill and Ergometric Bicycle}

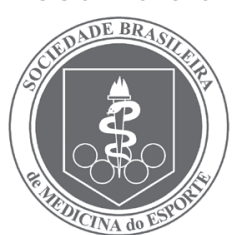

Artigo Original
Gerusa Eisfeld Milano

Neiva Leite

Núcleo de Pesquisa em Qualidade de Vida (NQV) - Universidade Federal do Paraná - Curitiba, PR.

\author{
Endereço para correspondência: \\ Gerusa Eisfeld Milano \\ Universidade Federal do Paraná \\ - UFPR \\ Departamento de Educação Física \\ Núcleo de Pesquisa em Qualidade \\ de Vida \\ Rua Coração de Maria, 92, \\ BR 116, km 95. \\ 80215-370 - Curitiba, PR - Brasil \\ E-mail: gerusamilano@hotmail.com
}

Submetido em 10/01/2008

Versão final recebida em 28/11/2008 Aceito em 08/01/2009

\begin{abstract}
RESUMO
O objetivo deste estudo foi investigar o consumo máximo de oxigênio $\left(\mathrm{VO}_{2 \max }\right)$ em adolescentes obesos e não obesos em esteira e bicicleta ergométrica. O estudo foi transversal e descritivo com a amostra de 54 indivíduos obesos (23 meninos e 31 meninas) e 33 não obesos (16 meninos e 17 meninas) com idade entre 10 e 16 anos. Utilizou-se o critério dos Centers for Disease Control and Prevention (CDC, 2000) para a classificação do índice de massa corporal (IMC). Foram avaliados o peso, estatura, índice de massa corporal (IMC) e IMC-escore Z. Para avaliação cardiorrespiratória foi realizado teste máximo em esteira e bicicleta ergométrica. Foram avaliados a frequência cardíaca máxima $\left(F C_{\max }\right)$, coeficiente respiratório ( $\left.\mathrm{RER}\right)$, consumo máximo de oxigênio $\left(\mathrm{VO}_{2 \max }\right)$, tempo total de teste (TT) e carga final (w) na bicicleta ergométrica. Utilizou-se o teste de $t$ de Student para a comparação dos ergômetros. Nas análises dos grupos, utilizou-se a ANOVA fatorial. Considerou-se significante $\mathrm{p}<0,05$. Os resultados revelaram que o peso, IMC e IMC-escore Z foram maiores nos obesos. Os valores de $V \mathrm{O}_{\text {max }}$ absoluto $\left(\mathrm{l} \cdot \mathrm{min}^{-1}\right.$ ) foram mais altos nos obesos, em ambos os gêneros, na esteira, sem diferenças entre os grupos na bicicleta. Entretanto, o $\mathrm{VO}_{2 \max }$ relativo ao peso corporal $\left(\mathrm{ml}^{\mathrm{kg}} \mathrm{kg}^{-1} \cdot \mathrm{min}^{-1}\right)$ foi menor no grupo obeso e nas meninas, em ambos os ergômetros. A carga de trabalho na bicicleta foi similar entre os obesos e não obesos. Concluiu-se que os obesos apresentaram valores de $\mathrm{VO}_{2 \max }$ mais baixos que os não obesos em ambos os ergômetros. Entretanto, o fato de o indivíduo obeso na bicicleta suportar a mesma carga final que o não obeso sugere-se que a bicicleta pode ser indicado como o melhor ergômetro para obesos.
\end{abstract}

Palavras-chave: obesidade, $\mathrm{VO}_{2 \text { max’ }}$ ergômetros.

\begin{abstract}
The aim of this study was to investigate the oxygen maximal uptake $\left(\mathrm{VO}_{2 \max }\right)$ in obese and non-obese adolescents on treadmill and ergometric bicycle. The study was transversal and descriptive with a sample of 54 obese individuals ( 23 boys and 31 girls) and 33 non-obese individuals (16 boys and 17 girls), mean age of 10-16 years. The criterion by the Centers for Disease Control and Prevention (CDC, 2000) was used for classification of body mass index (BMI). Weight, height, body mass index (BMI) and BMI-score Z were assessed. A maximal test on treadmill and ergometric bicycle was performed for cardiorespiratory assessment. Maximum heart rate $\left(H R_{\text {max }}\right)$, respiratory coefficient (REC), maximal oxygen uptake $\left(\mathrm{VO}_{2 \text { max }}\right)$, test total time $(\mathrm{TT})$ and final load ( $\mathrm{W}$ ) on ergometric bicycle were assessed. Student's t-test was used for ergometers comparison. Factorial ANOVA was used in the groups'analysis and $\mathrm{p}<0.05$ was considered significant. The results revealed that weight, BMI and BMl-score Z were higher in the obese subjects. The absolute $\mathrm{VO}_{2 \max }\left(\mathrm{ml}^{-1}\right)$ values were higher in the obese subjects in both genders on treadmill, with no differences between the bicycle group. However, the $\mathrm{VO}_{2 \max }$ concerned with body weight $\left(\mathrm{m}^{1} \mathrm{~kg}^{-1} \mathrm{~min}^{-1}\right)$ was lower in the obese group and in girls in both ergometers. The workload on bicycle was similar between obese and non-obese subjects. As a conclusion, the obese subjects presented $\mathrm{VO}_{2 \max }$ values lower than the non-obese ones in both ergometers. Nevertheless, the fact that obese subjects can work with the same final load of the nonobese subjects suggests that the bicycle can be considered as the best ergometer for obese individuals.
\end{abstract}

Keywords: obesity, $\mathrm{VO}_{2 \max }$ ergometers.

\section{INTRODUÇÃO}

O consumo máximo do oxigênio $\left(\mathrm{VO}_{2 \mathrm{max}}\right)$ é um dos melhores indicadores da aptidão cardiorrespiratória e um importante parâmetro preditivo de morbidades associadas ${ }^{(1)}$. Além de diagnosticar o nível de condicionamento cardiorrespiratório, a avaliação do $\mathrm{VO}_{2 \max }$ é também utilizada para acompanhamento e prescrição do treinamento aeróbio em atletas e sedentários ${ }^{(2)}$.

A escolha do ergômetro e protocolo é fundamental para o sucesso na avaliação do consumo máximo de oxigênio. Teoricamente, espera-se que os testes realizados em ergômetros diferentes apresentem valores máximos não coincidentes entre si. Usualmente, a esteira e a bicicleta ergométrica são os ergômetros mais utilizados, tanto em crianças como nos adultos, principalmente por reproduzir atividades habituais ${ }^{(3)}$. Na população em geral, são encontrados valores de $\mathrm{VO}_{2}$, em média, 10\% mais altos na esteira quando comparados com os da bicicleta ergométrica(4).

Na prática dos laboratórios clínicos brasileiros nota-se preferência pelos testes máximos na esteira em detrimento da bicicleta ergométrica; uma das razões alegadas são os maiores valores de $\mathrm{VO}_{2 \max }$ encontrados na esteira. Considerando as diferenças hemodinâmicas encontradas nos testes, sugere-se que estas sejam devidas ao efeito do peso corporal na sua realização e aos grupamentos musculares envolvidos ${ }^{(3)}$.

A sustentação ou não do peso é um fator relevante, principalmente em obesos, os quais possuem maior carga corporal a ser sustentada 
durante a avaliação do consumo máximo de oxigênio. Entretanto, na população obesa, existe discordância a respeito da avaliação de $\mathrm{VO}_{2 \max }$ na esteira e bicicleta. O estudo de Loftin et al. (2004) ${ }^{(5)}$ relatou não haver diferenças nos valores máximos entre os ergômetros, sugerindo que ambos podem ser utilizados em obesos. Entretanto, Maffeis et al. (1994)(6) encontraram diferença entre os ergômetros, com os maiores valores na esteira do que na bicicleta ergométrica. Dessa forma, nota-se não haver consenso entre as pesquisas de $\mathrm{VO}_{2 \max }$ em populações pediátricas que apresentam excesso de peso, revelando, assim, carência de dados que indiquem o melhor ergômetro para a avaliação em obesos.

O objetivo deste estudo foi comparar os parâmetros cardiorrespiratórios em adolescentes obesos e não obesos obtidos em testes máximos realizados em esteira e bicicleta ergométrica.

\section{MÉTODOS}

\section{Sujeitos}

Participaram do estudo 87 voluntários, com idade entre 10 e 16 anos, de ambos os gêneros, estudantes da rede pública de Curitiba e atendidos na Unidade de Endocrinologia Pediátrica da Universidade Federal do Paraná. Foram divididos em dois grupos de acordo com o índice de massa corporal (IMC) proposto pela classificação dos Centers for Disease Control and Prevention (CDC) ${ }^{(7)}$. O grupo obeso foi composto por 54 adolescentes (23 meninos e 31 meninas); o grupo não obeso, por 33 adolescentes (16 meninos e 17 meninas). Foram excluídos do estudo todos os indivíduos com sobrepeso, os pré-púberes e aqueles que apresentavam contra-indicações para a realização das avaliações. Todos os indivíduos e seus respectivos responsáveis preencheram um termo de consentimento, autorizando a avaliação e o uso dos seus dados. Esta pesquisa foi aprovada pelo Comitê de Ética em Pesquisa da Universidade Federal do Paraná, atendendo à Resolução 196/96.

\section{Instrumentos e procedimentos}

1) Avaliação antropométrica - A massa corporal foi mensurada utilizando uma balança antropométrica (Filizolaß) com resolução de $100 \mathrm{~g}$ e a estatura foi determinada com um estadiômetro com escala de $0,1 \mathrm{~cm}$. A partir dessas duas variáveis, calculou-se o IMC. Para o cálculo do IMC-escore Z, utilizou-se o IMC de cada indivíduo subtraído do valor correspondente ao $50^{\circ}$ percentil do IMC, dividido pelo desvio-padrão populacional, conforme valores disponibilizados pelo $\operatorname{CDC}^{(7)}$, para cada faixa etária e gênero.

2) Avaliação clínica - A avaliação foi realizada por um profissional da área de pediatria para detecção de eventuais doenças cardiovasculares nos participantes da pesquisa e para determinar o grau de maturação sexual(8).

3) Aptidão aeróbia ( $\mathrm{VO}_{2}$ máx) - A análise cardiorrespiratória mensurou variáveis respiratórias e metabólicas através das trocas gasosas com um sistema metabólico direto (Vista XT Metabolic System, EUA) computadorizado (Intel 486, DX2,66MHz), o qual forneceu informações acerca do consumo de oxigênio (VO2), produção de dióxido de carbono $\left(\mathrm{VCO}_{2}\right)$, ventilação pulmonar (VE), equivalentes respiratórios de oxigênio $\left(\mathrm{VE} / \mathrm{NO}_{2}\right)$ e gás carbônico $\left(\mathrm{VE} / \mathrm{VCO}_{2}\right)$ e razão de troca res- piratória $\left(\mathrm{RER}=\mathrm{VCO}_{2} / \mathrm{NO}_{2}\right)$. Foi utilizado um conjunto de máscara, gorro e turbina com vedação da boca e nariz levando o ar expirado ao equipamento de análise de gases. Para a monitoração da frequência cardíaca foi utilizado um frequencímetro cardíaco (Polar - A1).

Considerou-se $\mathrm{VO}_{2 \max }$ quando pelo menos dois dos seguintes critérios foram observados: a) exaustão ou inabilidade para manter a velocidade requerida; b) RER > 1,0; c) FC > 190bpm. Utilizou-se a média dos três maiores valores seguidos para o estabelecimento do $\mathrm{VO}_{2 \max }{ }^{(9)}$.

Na avaliação realizada em bicicleta ergométrica, utilizou-se o protocolo de McMaster ${ }^{(10)}$, a cadência foi de $60 \mathrm{rpm}$, com a carga inicial de 25 watts (W), com aumento progressivo de carga em $25 \mathrm{~W}$ para as meninas e meninos com estatura $<1,60 \mathrm{~m}$ e em $50 \mathrm{~W}$ para meninos com estatura $\geq 1,60 \mathrm{~m}$, a cada dois minutos até o esforço máximo. Na esteira ergométrica, o protocolo utilizado foi o de Balke modificado, mantendo a velocidade fixa em 3,25mph e inclinação de 6\%, com incremento de $2 \%$ a cada três minutos, até o esforço máximo(11). Entre os testes respeitou-se o período mínimo de 24h e máximo de uma semana, sendo o primeiro realizado em esteira e o segundo em bicicleta ergométrica.

\section{Análise estatística}

Os dados foram apresentados através de estatísticas descritivas, tabelas e gráficos. Utilizou-se o teste de $t$ de Student para dados pareados na comparação das médias aritméticas entre os ergômetros. Nas análises entre os grupos, utilizou-se a ANOVA fatorial. Realizaram-se as análises por meio do programa estatístico Statistica 6.0, considerando nível de significância de 5\% ( $<<0,05)$. Para o cálculo da amostra o número de participantes foi calculado para um poder estatístico (erro $\beta$ ) de 0,87; totalizando assim, no mínimo, 16 participantes para cada grupo e gênero.

\section{RESULTADOS}

Este estudo avaliou os valores de $\mathrm{VO}_{2 \max }$ em adolescentes obesos e não obesos na esteira e bicicleta ergométrica. As meninas e os meninos do grupo obeso apresentaram peso, IMC e IMC-escore Z maiores do que os do grupo não obeso ( $p<0,0000$ ), consequência da própria caracterização da amostra estudada. Os meninos obesos apresentaram idade menor do que os não-obesos; nas meninas não foram encontradas diferenças. Todos os indivíduos eram púberes.

O IMC das meninas obesas foi maior do que o dos meninos obesos ( $p=0,006)$, mas sem diferenças na estatura e no IMC-escore Z. No grupo não obeso não houve diferenças no IMC e estatura entre os gêneros. Os valores médios e desvios-padrões (DP) das características gerais dos indivíduos estudados constam na tabela 1.

Todos os avaliados realizaram o teste cardiorrespiratório máximo em esteira e bicicleta ergométrica. Os critérios preestabelecidos como parâmetros máximos, a $F C_{\max }$ e o RER não diferiram entre os gêneros e grupos, em ambos os ergômetros. O tempo médio para duração do teste na esteira foi maior nos meninos do grupo não obeso $(p<0,05)$ do que no obeso; na bicicleta, não houve diferença entre os grupos. A carga final obtida no teste em bicicleta foi similar entre os grupos, os meninos apresentando maiores valores do que as meninas ( $p<0,000$ ) (tabela 2).

Os valores médios do $\mathrm{VO}_{2 \max }$ absoluto foram maiores nos obesos do que nos não obesos na esteira; não foram encontradas diferenças

Tabela 1. Média e desvio-padrão da idade, peso, estatura (est), IMC e IMC-escore Z

\begin{tabular}{|c|c|c|c|c|c|c|}
\hline \multirow[b]{2}{*}{ Variáveis } & \multicolumn{3}{|c|}{ Meninos } & \multicolumn{3}{|c|}{ Meninas } \\
\hline & Não obeso $(\boldsymbol{n}=16)$ & Obeso $(\boldsymbol{n}=23)$ & $\mathrm{p}$ & Não obesas $(\boldsymbol{n}=17)$ & Obesas $(n=31)$ & $\mathrm{p}$ \\
\hline Idade (anos) & $14,14 \pm 1,47$ & $12,81 \pm 1,61$ & $p<0,05$ & $13,81 \pm 1,95$ & $13,57 \pm 1,70$ & ns \\
\hline Peso (kg) & $54,94 \pm 9,58$ & $76,94 \pm 14,37$ & $p<0,00$ & $45,95 \pm 7,62$ & $83,83 \pm 12,41$ & $p<0,00$ \\
\hline Est $(\mathrm{cm})$ & $168,3 \pm 9,10$ & $161,51 \pm 10,4$ & ns & $156,3 \pm 9,40$ & $161,13 \pm 6,01$ & ns \\
\hline $\operatorname{IMC}\left(\mathrm{kg} / \mathrm{m}^{2}\right)$ & $19,27 \pm 1,83$ & $29,32 \pm 3,61$ & $p<0,00$ & $18,70 \pm 1,82$ & $32,21 \pm 3,86$ & $P<0,00$ \\
\hline IMC Esc Z & $-0,10 \pm 0,72^{c}$ & $3,37 \pm 1,4$ & $p<0,00$ & $-0,26 \pm 0,55$ & $3,63 \pm 1,23$ & $P<0,00$ \\
\hline
\end{tabular}




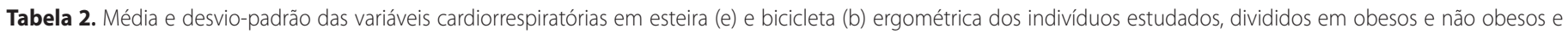
de acordo com o sexo

\begin{tabular}{|c|c|c|c|c|c|c|}
\hline \multirow{2}{*}{$\begin{array}{c}\text { Grupos } \\
\text { Variáveis }\end{array}$} & \multicolumn{3}{|c|}{ Meninos } & \multicolumn{3}{|c|}{ Meninas } \\
\hline & Não obeso $(\boldsymbol{n}=16)$ & Obeso $(\boldsymbol{n}=23)$ & $\mathrm{p}$ & Não obesa $(\boldsymbol{n}=17)$ & Obesa $(\boldsymbol{n}=31)$ & $\mathrm{p}$ \\
\hline$F C_{\text {Max }}$ Est & $196,50 \pm 11,15$ & $194,40 \pm 10,42$ & $\mathrm{~ns}$ & $197 \pm 8,62$ & $192 \pm 10,18$ & $\mathrm{~ns}$ \\
\hline $\mathrm{FC}_{\text {Max }} \mathrm{BiC}$ & $187 \pm 16,14$ & $183,27 \pm 15,12$ & ns & $189,5 \pm 7,68$ & $182,19 \pm 10,16$ & ns \\
\hline RER Est & $1,00 \pm 0,05$ & $1,00 \pm 0,1$ & ns & $0,98 \pm 0,03$ & $1,01 \pm 0,09$ & ns \\
\hline RER Bic & $1,02 \pm 0,12$ & $0,98 \pm 0,04$ & ns & $0,99 \pm 0,07$ & $1,00 \pm 0,07$ & ns \\
\hline $\mathrm{VO}_{2 \max }(1 . \mathrm{min})^{1} \mathrm{Est}$ & $2,61 \pm 0,72^{c}$ & $2,77 \pm 0,91$ & $p<0,05$ & $2,00 \pm 0,24^{b}$ & $2,80 \pm 0,69$ & $p<0,05$ \\
\hline $\mathrm{VO}_{2 \max }(\mathrm{I} \cdot \mathrm{min})^{-1} \mathrm{Bic}$ & $2,51 \pm 0,66$ & $2,57 \pm 0,95$ & $\mathrm{~ns}$ & $1,82 \pm 0,25$ & $2,28 \pm 0,40$ & $\mathrm{~ns}$ \\
\hline $\mathrm{VO}_{2 \max } \mathrm{ml} \cdot \mathrm{kg}^{-1} \cdot \mathrm{min}^{-1} \mathrm{Est}$ & $51,69 \pm 5,96^{a c}$ & $36,88 \pm 5,62$ & $p<0,00$ & $41,05 \pm 4,23^{b}$ & $32,35 \pm 5,1$ & $p<0,00$ \\
\hline $\mathrm{VO}_{2 \max } \mathrm{ml} \cdot \mathrm{kg}^{-1} \cdot \mathrm{min}^{-1} \mathrm{BiC}$ & $50,33 \pm 7,86^{a c}$ & $32,63 \pm 6,88$ & $p<0,00$ & $39,45 \pm 5,33^{b}$ & $26,84 \pm 4,22$ & $p<0,00$ \\
\hline TT (min) Est & $20,10 \pm 5,74^{a c}$ & $13,43 \pm 3,80$ & $p<0,05$ & $14,84 \pm 4,08$ & $10,44 \pm 3,10$ & $p<0,05$ \\
\hline $\mathrm{TT}$ (min) Bic & $8,79 \pm 1,56$ & $8,84 \pm 1,56$ & ns & $8,65 \pm 1,23$ & $10,29 \pm 1,73$ & ns \\
\hline Carga (W) Bic & $160 \pm 42,82^{a}$ & $131,48 \pm 49,80$ & ns & $123,18 \pm 6,81$ & $116,91 \pm 26,63$ & ns \\
\hline
\end{tabular}

entre os gêneros. Na bicicleta, os meninos apresentaram maiores valores médios de $\mathrm{VO}_{2 \max }$ absoluto do que as meninas $(\mathrm{p}<0,00)$, sem diferenças entre os obesos e não obesos. Os valores médios e desviospadrões (DP) das variáveis cardiorrespiratórias obtidas no repouso e teste máximo constam na tabela 2.

O grupo obeso apresentou maiores valores de $\mathrm{VO}_{2 \max } \mathrm{ml} \cdot \mathrm{kg}^{-1} \cdot \mathrm{min}^{-1}$ obtidos na esteira do que na bicicleta ergométrica em ambos os gêneros ( $p<0,000$ ); entretanto, no grupo não obeso, apenas as meninas apresentaram maiores valores na esteira ( $p<0,05)$; nos meninos não foram encontradas diferenças entre os ergômetros (figura 1).

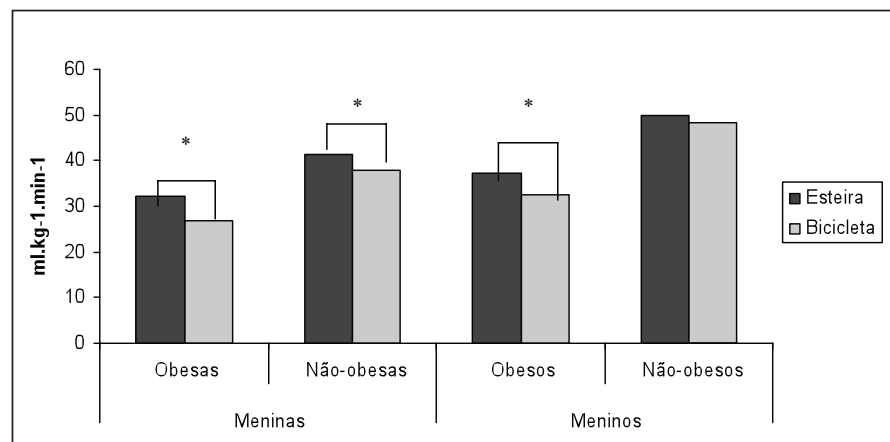

Figura 1. Valores médios de $\mathrm{VO}_{2 \max }\left(\mathrm{ml}_{\mathrm{kg}} \mathrm{kg}^{-1} \cdot \mathrm{min}^{-1}\right)$ em esteira e bicicleta ergométrica nos indivíduos obesos e não obesos.

* Diferença entre ergômetros $p<0,05$

\section{DISCUSSÃO}

A primeira proposta deste estudo foi avaliar os $\mathrm{VO}_{2 \max }$ obtidos na esteira e bicicleta ergométrica em adolescentes obesos e não obesos. Nos estudos que envolvem adolescentes, um fator relevante e que deve ser observado é o estágio maturacional, porque há íntima relação entre os valores de $\mathrm{VO}_{2 \max }$ e a fase puberal(12). Especialmente em indivíduos obesos, a avaliação do estágio maturacional é mais importante do que a idade cronológica. Wang (2002) ${ }^{(13)}$ revelou que os adolescentes com excesso de peso apresentam tendência à maturação mais precoce quando comparados com os não obesos. Isso provavelmente ocorreu neste estudo, em que todos os indivíduos eram púberes, porém a idade cronológica foi menor nos meninos obesos do que nos não obesos.

A variável $F C_{\max }$ depende da idade cronológica ${ }^{(14)}$. Alguns autores ${ }^{(15-16)}$ sugerem que a $\mathrm{FC}_{\max }$ também sofre influência pela obesidade. Estudo que comparou meninas obesas e não obesas encontrou valores de $\mathrm{FC}_{\max }$ na esteira ergométrica menores naquelas (192 \pm 9bpm) do que nestas
$(203 \pm 8 \mathrm{bpm})^{(15)}$. Resultados semelhantes foram encontrados no trabalho de Norman et al. (2005) (16), no qual adolescentes obesos (186 \pm 13 e $175 \pm$ 18bpm) também apresentavam menores valores do que os não obesos (196 \pm 11 e $197 \pm 17 \mathrm{bpm})$, em teste de bicicleta e corrida de 12 minutos.

No entanto, outras pesquisas observaram semelhanças na $\mathrm{FC}_{\max }$ obtidas em adolescentes obesos e não obesos, tanto na esteira ${ }^{(17-19)}$, como na bicicleta ergométrica ${ }^{(5)}$. No presente estudo, não foram encontradas diferenças significativas nas respostas fisiológicas da $\mathrm{FC}_{\max }$ entre os grupos obesos e não obesos em ambos os ergômetros. Talvez a diferença na idade cronológica entre os meninos obesos e não obesos tenha minimizado as diferenças na $\mathrm{FC}_{\max }$ entre os grupos.

Além de os estudos diferirem quanto à existência ou não de diferenças na $F C_{\max }$ entre obesos e não obesos, existe discordância a respeito dos valores de $F C_{\max }$ entre os ergômetros. Alguns trabalhos relataram valores similares na esteira e bicicleta ergométrica ${ }^{(6,20)}$; contudo, outros encontraram maiores valores na esteira(21). Neste estudo, quando comparada a FC $_{\max }$ obtida nos ergômetros, os testes na esteira ergométrica apresentaram maiores valores do que na bicicleta. Outro parâmetro importante e que também apresenta grande flutuação junto com a FC max é o RER. No estudo de Loftin et al. (2004)(6), os valores do RER foram similares na esteira $(0,93 \pm 0,05)$ e bicicleta ergométrica $(1,01 \pm 0,08)$. Em crianças, o estudo de Goran et al. (2000)(22) mostrou valores similares entre obesas $(1,03 \pm 0,05)$ e não obesas $(1,02 \pm 0,07)$. Corroborando esses estudos, os valores de RER não diferiram entre os ergômetros na presente pesquisa, em gêneros e grupos.

Alguns autores sugerem a utilização do valor de platô de $\mathrm{VO}_{2}$ para considerar o valor de $\mathrm{VO}_{2 \text { max }}$ porém, recentes investigações têm observado a dificuldade de ocorrer esse fenômeno em $\operatorname{crianças~}^{(23-24)}$, sedentários ${ }^{(25)}$ e indivíduos com doenças ${ }^{(26)}$. Alguns autores têm utilizado o valor médio dos três maiores valores seguidos como $\mathrm{VO}_{2 \max }$ de cada indivíduo(11).

$\mathrm{O} \mathrm{VO}_{2 \text { max }}$ em valores absolutos está relacionado diretamente com o tamanho corporal; dessa forma, pesquisas relatam maiores valores em adolescentes obesos do que nos não obesos ${ }^{(5,16,19)}$; outros encontraram valores semelhantes entre os grupos ${ }^{(17,27)}$. Segundo Ekelund et al. (2004) (19), o fato de o indivíduo obeso apresentar valores de $\mathrm{VO}_{2 \max }$ absoluto mais elevados ou semelhantes aos dos não obesos indica capacidade funcional preservada, pois a equação de Fick (que relaciona o oxigênio bombeado pelo coração e o captado pelo tecidos) revela que o coração parece fornecer oferta adequada de oxigênio para o tecido muscular. Porém, quando expressos relativos ao peso corporal, os obesos, por ter maior massa corporal, apresentam menores valores do que os não obesos $(5,6,17,18,19,22,27)$.

Ao comparar os gêneros, os resultados deste presente estudo corroboram a literatura(12) que reporta maiores valores de $\mathrm{VO}_{2 \max }$ em meninos que em meninas. 
Harmansen e Satlin (1969)(4) compararam o $\mathrm{VO}_{2 \text { max }}$ relativo ao peso corporal em esteira e bicicleta ergométrica; encontraram que, em média, os valores de $\mathrm{VO}_{2 \max }$ são 10\% maiores na esteira do que na bicicleta; essas diferenças foram atribuídas à fadiga muscular localizada e à menor massa muscular envolvida. O estudo de Maffeis et al. (1994)(6) obteve maior diferença nos valores de $\mathrm{VO}_{2 \max }$ relativo, entre obesos e não obesos, na bicicleta do que na esteira ergométrica. Entretanto, Loftin et al. (2004)(5) relataram ter encontrado valores de $\mathrm{VO}_{2 \max }$ relativos em obesos semelhantes, tanto na esteira como na bicicleta ergométrica, indicando que ambos os ergômetros podem ser utilizados na avaliação, o que revela haver discordância na literatura quanto à avaliação de obesos em diferentes ergômetros.

No presente estudo, o grupo obeso apresentou $\mathrm{VO}_{2 \max }$ em valor absoluto superior ao do não obeso no teste em esteira, sem diferenças

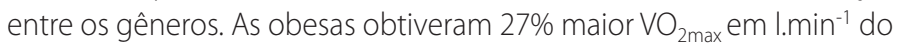
que as não obesas e a diferença foi de $23,52 \%$ para os meninos obesos em relação aos não obesos. Entretanto, na bicicleta, o $\mathrm{VO}_{2 \text { max }}$ absoluto não diferiu entre os grupos; foram observadas diferenças apenas entre os gêneros, os meninos apresentando maiores valores do que as meninas. As semelhanças do $\mathrm{VO}_{2 \max }$ absoluto em bicicleta ergométrica entre obesos e não obesos podem ser justificadas pela sustentação do corpo durante o teste. Na esteira, o obeso, por suportar a massa corporal na execução do teste, apresenta maior sobrecarga para uma mesma velocidade e inclinação, refletindo em maior valor de $\mathrm{VO}_{2 \max }$ absoluto.

Esta pesquisa corroborou o estudo de Maffeis et al. (1994)(6), pois, ao comparar o $\mathrm{VO}_{2 \max }$ em valores relativos ao peso corporal, as meninas não obesas apresentaram maior valor de $\mathrm{VO}_{2 \max }$ em ambos os ergômetros, sendo maior diferença na bicicleta $(23,79 \%)$ do que na esteira ergométrica (18,31\%) em relação às meninas obesas. Nos meninos não obesos os valores de $\mathrm{VO}_{2 \max }$ relativos foram $31,63 \%$ maiores no teste em bicicleta e $25,56 \%$ na esteira do que os meninos obesos. As meninas não obesas também apresentaram maiores valores de $\mathrm{VO}_{2 \max }$ relativo ao peso corporal na esteira em relação à bicicleta ergométrica. Somente os meninos do grupo não obeso apresentaram semelhanças entre os ergômetros em relação ao $\mathrm{VO}_{2 \max }$ relativo ao peso corporal.

Nesta pesquisa, a ausência de diferenças entre o $\mathrm{VO}_{2 \max }$ na esteira e bicicleta nos meninos não obesos pode ser resultado do protocolo utilizado na esteira, o qual ocasionou umas das limitações do estudo. O teste foi o mesmo para todos os grupos, o qual provocou um tempo longo de teste para os meninos não obesos. Provavelmente, ocasionaram valores de $\mathrm{VO}_{2 \text { max }}$ mais baixos na esteira, visto que o tempo recomendado é em média de $10 \pm 2$ minutos ${ }^{(28)}$. Mas o tempo do teste maior dos não obesos foi inevitável, pois o protocolo neste estudo foi escolhido de acordo

\section{REFERÊNCIAS BIBLIOGRÁFICAS}

1. American College of Sports Medicine ACSM'S Resource Manual for Guidelines for Exercise Testing and Prescription. $5^{\text {th }}$ ed. Philadelphia: Lippincott Williams \& Wilkins, 2006.

2. Wilmore JH, Costill DL, editors. Fisiologia do Exercício. 1 ed. Săo Paulo: Manole; 2001.

3. Neder JA, Nery LE, editors. Fisiologia clínica do exercício: teoria e prática. 1 ed. São Paulo: Artes Médicas; 2003.

4. Harmansen L, Saltin B. Oxygen uptake during maximal treadmill and bicycle exercise. J Appl Physiol 1969; $26(1): 31-7$

5. Loftin M, Sothern M, Warren B, Udall J. Comparison and VO2 peak during treadmill and cycle ergometry in severely overweight youth. JSSM 2004; 3:254-60.

6. Maffeis C, Schena F, Zaffanello M, Zoccante L, Schultz Y, Pinelli L. Maximal aerobic power during running and cycling in obese and non-obese children. Acta Paediatr 1994; 83:223-226.

7. Kuczmarski RJ, Ogden CL, Guo SS, Grummer-Strawn LM, et al. CDC growth charts: United States. Advance data from vital and health statistics; n. 314. Hyattsville, Maryland: National Center for Health Statistics, 2000.

8. Tanner JM. Normal growth and techniques of growth assessment. Clin Endocrinol Metab 1986; 15(3): 411-451.

9. Leite N. Obesidade Infanto-juvenil: efeito da atividade física e da orientação nutricional sobre a resistência a insulina. Tese de doutorado Universidade Federal do Paraná. 2005.

10. Bar-Or O. Pediatric Sports Medicine for the Practitioner. New York: Springer-Verlag, p. 315-338, 1983.

11. Rowland TW. Exercise and children's health. Champaign: Human Kinetics Books, 1990.

12. Geithner CA, Thomis MA, Eynde B, Maes H, Loos R, Peeters M, Claessens A, et. al. Growth in Peak Aerobic Power during Adolescence. Med. Sci. Sports Exerc 2004; 36 (9):1616-24.

13. Wang Y. Is Obesity Associated With Early Sexual Maturation? A Comparison of the Association in American Boys Versus Girls. Pediatrics 2002; 110:903-10.

14. Tanaka H, Monahan KD, Seals DR. Age-Predicted Maximal Heart Rate Revisited. JACC 2001; 37(1): 153-6.

15. Loftin M, Sothern M, Vanvrancken C, O'hanlon A, Udall J. Effect of obesity status on heart rate peak in female youth. Clin Pediatr 2003; 42:505-10. com as recomendações para a população pediátrica obesa ${ }^{(10)}$. Contudo, mesmo com essa limitação, os indivíduos não obesos apresentaram maiores valores de $\mathrm{VO}_{2 \max }$ relativo ao peso corporal do que os obesos. O estudo de Maffeis et al. (1994)(6), que comparou obesos e não obesos, também utilizou o mesmo protocolo para ambos os grupos. Porém, mesmo com maior tempo de teste, todos os grupos alcançaram os critérios preestabelecidos como parâmetros máximos.

Apesar de a bicicleta ergométrica apresentar os menores valores de $\mathrm{VO}_{2 \max }\left(\mathrm{ml} \mathrm{kg}^{-1} \cdot \mathrm{min}^{-1}\right)$ para indivíduos obesos, os valores de $\mathrm{VO}_{2 \max }$ absoluto e a carga de trabalho foram similares entre os grupos. Provavelmente porque na bicicleta ergométrica, com a sustentação do corpo no selim, houve a minimização da influência da massa corporal na avaliação da aptidão cardiorrespiratória, permitindo um teste máximo com cargas similares entre grupos obesos e não obesos.

Portanto, os resultados deste estudo indicaram que o teste cardiorrespiratório apresentou qualidade considerável, todos os avaliados atingindo os critérios para $\mathrm{VO}_{2 \max }$ de acordo com o proposto na literatura. Embora a amostra deste estudo seja por conveniência, ela representa os valores para o grupo investigado, porém, nossos dados não devem ser extrapolados para toda a população obesa. Sugerem-se novos estudos que utilizem diferentes protocolos como, por exemplo, o de rampa e com amostras em diferentes faixas etárias.

\section{CONCLUSÕES}

A comparação entre os ergômetros neste estudo revelou que no grupo obeso e nas meninas não obesas os valores médios de $\mathrm{VO}_{2 \text { max }}$ foram superiores na esteira em relação à bicicleta ergométrica. No entanto, ao comparar a carga de trabalho máxima alcançada no teste em bicicleta, verificou-se que há equivalência das atividades entre obesos e não obesos, provavelmente pela característica do ergômetro em sustentar a massa corporal, através do selim. A bicicleta foi um ergômetro que facilitou a execução do teste em indivíduos obesos, sugerindo assim que, talvez, o obeso apresentasse maiores condições de executar os exercícios físicos e, consequentemente, proporcionasse maior adesão ao treinamento, quando o ergômetro não necessita suportar o peso corporal durante a atividade. Sugerem-se novos estudos que avaliem $\mathrm{VO}_{2 \max }$ de indivíduos obesos em diferentes ergômetros, faixas etárias e verificando a influência da massa corporal através do método alométrico.

Todos os autores declararam não haver qualquer potencial conflito de interesses referente a este artigo.

16. Norman AC, Drinkard B, Mcduffie JR, Ghorbani S, Yanoff LB, Yanovski JA. Influence of Excess Adiposity on Exercise Fitness and Performance in Overweight Children and Adolescents. Pediatrics 2005; 115:690-6.

17. Zanconato S, Baraldi E, Santuz P, Rigon F, Vido L, Dalt LD, et al. Gas exchange during exercise in obese children. Eur J Pediatr 1989, 148: 614-7.

18. Marinov B, Kostianev S, Turnoska T.Ventilatory efficiency and rate of perceived exertion in obese and nonobese performing standardized exercise. Clin Physiol Funct Imagig 2002; 22(4): 254-260.

19. Ekelund U, Franks P, Wareham N, Âman J. Oxygen uptakes adjusted for body composition in normal-weight and obese adolescents. Obes Res 2004; 12(3): 513-520.

20. Araújo CG, Pinto VLM. Frequência cardíaca máxima em testes de exercício em esteira rolante e em cicloergômetro de membros inferiores. ABC 2005; 85(1):45-9,

21. Turley KR, Wilmore JH. Cardiovascular responses to treadmill and cycle ergometer exercise in children and adults. J Appl Physiol 1997; 83(3): 948-957.

22. Goran M, Fields DA, Hunter GR, Herd SL, Weinsier RL. Total body fat does not influence maximal aerobic capacity. Int J Obes 2000; 24:841-848.

23. Paridon SM, Alpert BS, Boas SR, Cabrera ME, Caldarera LL, Daniels SR, et al. Clinical stress testing in the pediatric age group a statement from the american heart association council on cardiovascular disease in the young, committee on atherosclerosis, hypertension, and obesity in youth. Circulation 2006; 113:1905-20.

24. Karila C, Blic J, Waernessyckle S, Benoist M, Scheinmann P. Cardiopulmonary Exercise Testing in Children An Individualized Protocol for Workload Increase. Chest 2001; 120 (1):81-7

25. Neder JÁ, Nery LE. Teste de Exercício Cardiopulmonar. Jornal de Pneumologia 2002; 28 supl.3.

26. Rowland TW, Cunningham LN. Oxygen uptake plateau during maximal treadmill exercise in children. Chest 1992; 101:485-9.

27. Loftin M, Sotheen M, Trodclair L, O'hanlon A, Miller J, Udall J. Scalin VO2 peak in obese and non-obese girl. Obes Rev 2001; 9: 290-6.

28. Buchfuhrer Mj, Hansen JE, Robinson TE, Sue D, Wasserman K, Whipp B. Optimizing the exercise protocol for cardiopulmonary assessment. J Appl Physiol 1983; 55(5):1558-64. 